\title{
Recurrent maxillary sinus cancer with only adrenal metastasis
}

\author{
HYE JUNG CHANG ${ }^{1}$, JOON-YOUNG HUR $^{2}$, KYU YEOUN WON $^{3}$, BOKSOON CHANG $^{4}$ and HA YEON LEE \\ ${ }^{1}$ Division of Hematology-Oncology, Department of Internal Medicine; ${ }^{2}$ Department of Internal Medicine, \\ Graduate School of Medicine, Kyung Hee University Hospital at Gandgong; Departments of ${ }^{3}$ Pathology and \\ ${ }^{4}$ Pulmonary and Critical Care Medicine, Kyung Hee University Hospital at Gandgong, Seoul, Republic of Korea
}

Received February 28, 2017; Accepted August 1, 2017

DOI: $10.3892 / \mathrm{mco} .2017 .1427$

\begin{abstract}
Maxillary sinus cancer is rare, and often presents as a locally advanced disease. Recurrence commonly occurs locoregionally, while fewer patients present with distant metastasis; the most common sites involved are the lung and bone. This report discusses the case of a 64-year-old male who presented with a mass in the left submandibular area. Biopsy was performed and histological analysis identified a poorly differentiated squamous cell carcinoma. After staging work up, it was concluded the patient had a maxillary sinus squamous cell carcinoma at clinical stage IVA. The patient received a left partial maxillectomy and left radical neck dissection followed by postoperative chemoradiotherapy. After three months of CRT, the patient developed a left adrenal gland metastasis without locoregional failure. The patient subsequently received a laparoscopic left adrenalectomy and the results of histological analysis revealed a poorly-differentiated squamous cell carcinoma. Within one month of surgery, the patient had developed multiple metastases at the left adrenalectomy site and succumbed to the disease four months later.
\end{abstract}

\section{Introduction}

Maxillary sinus cancer is rare and often presents as a locally advanced disease (1). Recurrence commonly occurs locoregionally, although a minority of patients may experience distant metastasis, and the most commonly involved sites are the lungs and bone $(2,3)$. The current report discusses the case of a 64-year-old male who presented with a mass in the left submandibular area at the Kyung Hee University Hospital at Gangdong in November 2013. Biopsy was performed and histological analysis identified a poorly differentiated

Correspondence to: $\mathrm{Dr} \mathrm{Ha}$ Yeon Lee, Division of Hematology-Oncology, Department of Internal Medicine, Graduate School of Medicine, Kyung Hee University Hospital at Gangdong, 892 Dongnam-ro, Gangdong-gu, Seoul 05278, Republic of Korea E-mail: golhy01@naver.com

Key words: maxillary sinus neoplasms, adrenal glands, salvage therapy squamous cell carcinoma. A staging workup was conducted, including chest X-ray, paranasal sinus computed tomography (CT), neck CT, chest CT and positron emission tomography (PET)-CT. After staging workup, it was concluded the patient had a maxillary sinus squamous cell carcinoma at clinical stage IVA, which was classified according to the American Joint Committee on Cancer (AJCC) staging system (4). The patient received a left partial maxillectomy and a left radical neck dissection, followed by postoperative chemoradiotherapy (CRT). After three months of CRT, the patient developed a left adrenal gland metastasis without locoregional failure. The patient received a laparoscopic left adrenalectomy and histological analysis revealed it to be a poorly-differentiated squamous cell carcinoma. Within one month of surgery, the patient developed multiple metastases at the left adrenalectomy site, and succumbed to the disease four months later.

Paranasal sinus cancer is a rare type of tumor, representing $\sim 3 \%$ of head and neck malignancies (1). The most common site of these tumors is the maxillary sinus (5). Patients with maxillary sinus cancer tend to be asymptomatic until the tumor invades the adjacent structures; therefore, they often present with locally advanced disease (6).

Tumor recurrence commonly occurs within one year of diagnosis, with locoregional failure (3). Relatively few patients present with distant metastasis, for which the lungs and bone are the most commonly involved sites (3). This report presents a rare case of recurrent maxillary sinus cancer with only adrenal gland metastasis.

\section{Case report}

A 64-year-old male presented with pain in the left submandibular area and was treated with a three-week course of antibiotics at the local clinic. However, the patient continued to experience painful swelling and tenderness in the left submandibular area and was eventually admitted to the Kyung Hee University Hospital at Gangdong in November 2013. The patient had no significant medical history. Waters' view X-ray revealed a haziness on the left maxillary sinus and bone resorption on the left buccal vestibular region of no. 26. The patient had experienced tenderness at the left buccal vestibular region of no. 26 and the left submandibular region. The initial diagnosis was an odontogenic sinusitis through the dental caries on the palatal root of no. 26. Tooth no. 26 was extracted and the patient was prescribed oral antibiotics. Although the 

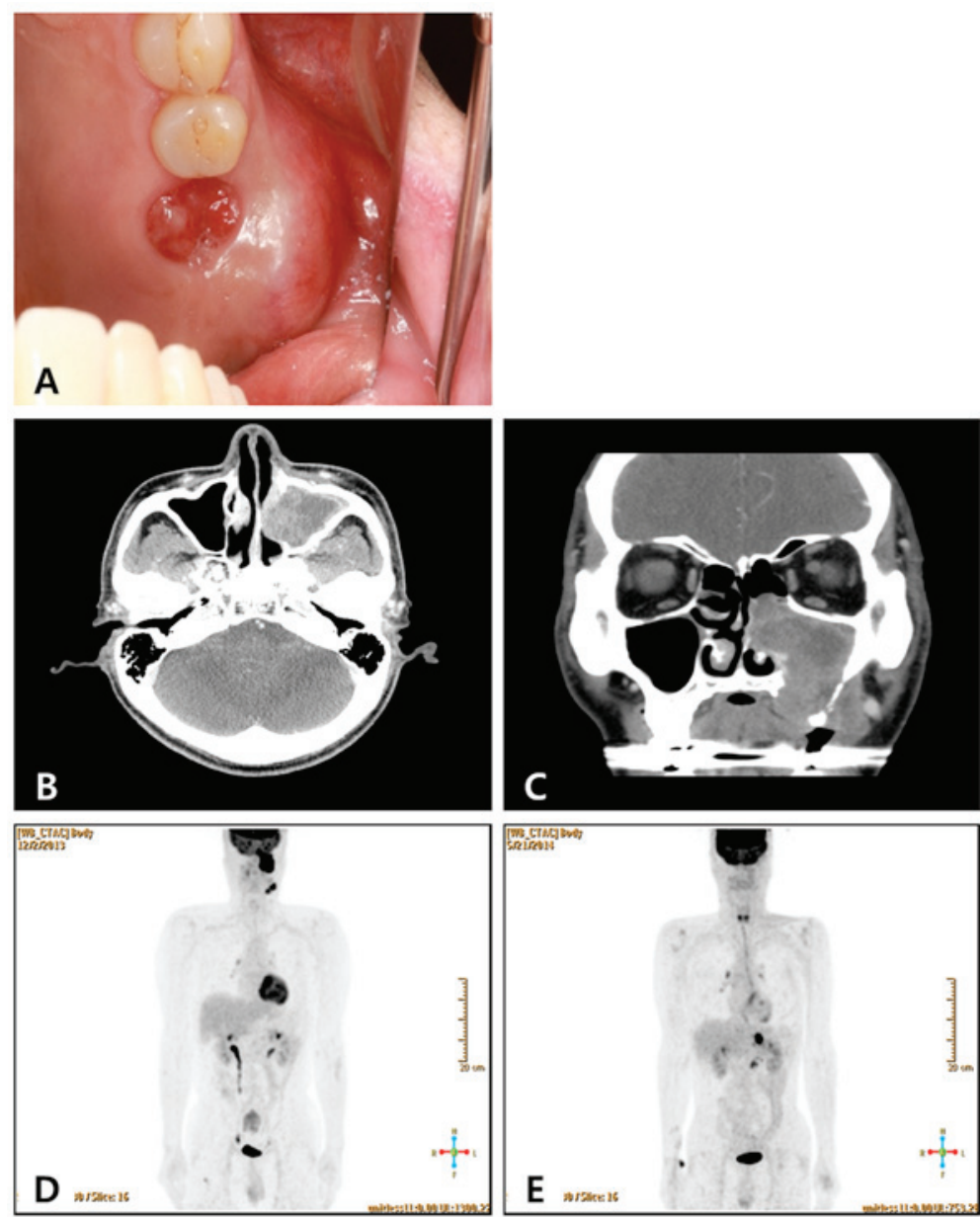

Figure 1. Serial images obtained via neck CT and PET/CT. (A) Exophytic granulation tissue was observed at the tooth no. 26 extraction site. (B) CT revealed destruction of the lateral wall of the maxillary sinus antrum and inferior wall, and tumor invasion to the left alveolar process of maxilla. (C) Invasion extension to the left buccal space and the left oral cavity. (D) PET/CT indicated that lesions involving the left maxillary alveolar process area, nasal cavity and two lymph nodes in the left submandibular area. (E) PET/CT revealed a hypermetabolic lesion in the left adrenal gland three months following the completion of CRT. CT, computed tomography; PET, positron emission tomography; CRT, chemoradiotherapy.
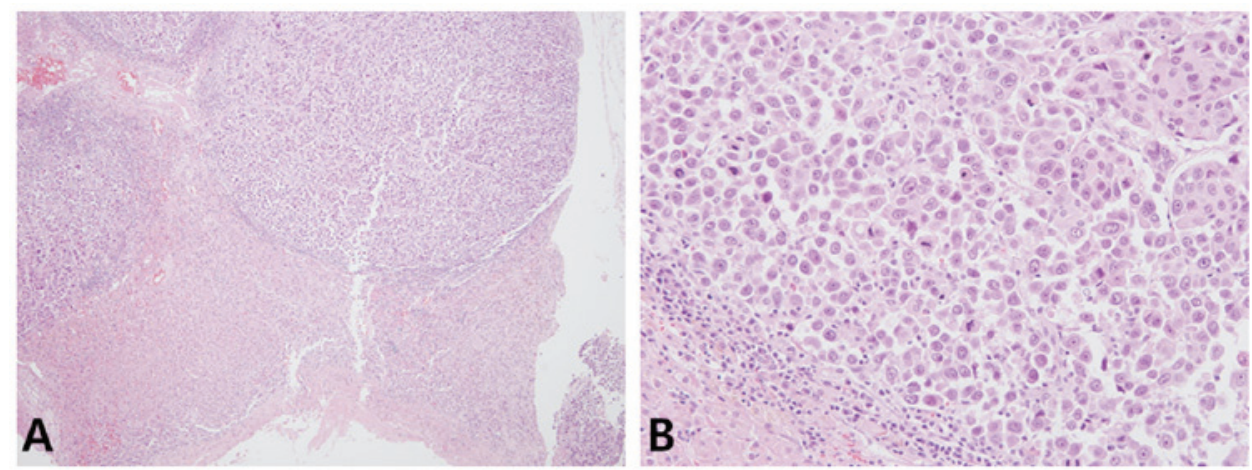

Figure 2. Microscopic findings in the resected adrenal gland tissue. (A) Multiple metastatic nodules (upper part of the figure) were present in the adrenal gland. (Hematoxylin and eosin stain, x20). (B) Magnified view indicated poorly-differentiated squamous cell carcinoma without formation of keratin pearls (hematoxylin and eosin stain, $\mathrm{x} 400$ ).

patient used the antibiotics for three weeks, they were still experiencing painful swelling in the left submandibular area, which had extended to the left maxillary sinus. Intraoral examination revealed a granulation tissue was protruding at the tooth no. 26 extraction site, and a biopsy at that site was subsequently performed (Fig. 1A).
Histological examination of the biopsy tissue sample revealed a poorly-differentiated squamous cell carcinoma. CT showed destruction of the left lateral maxillary sinus wall, invasion of the nasal cavity, destruction of the maxilla's medial inferior wall as well as destruction of the left oral cavity. In addition, enlarged lymph nodes in the left neck at level IB 
and level IIA were observed. A PET/CT scan was performed, and the lesion was determined to be confined to the primary site and the ipsilateral neck lymph nodes (Fig. 1B-D). After a staging workup, including CT and PET/CT, it was concluded that the patient had a maxillary sinus squamous cell carcinoma at clinical stage IVA (cT3N2), according to the AJCC staging system (4). The patient was then referred to the maxillofacial surgeon and received a left partial maxillectomy and ipsilateral radical neck dissection. The pathological findings indicated poorly-differentiated squamous cell carcinoma with neck lymph nodes metastasis (9/30). Negative resection margins were achieved but the safety margin was close; the patient received adjuvant concurrent CRT.

One month following the completion of CRT, a neck CT scan was used to assess the primary tumor status. There was no visible evidence of locoregional failure. After two months of CRT, the tumor status was re-examined using neck CT and chest CT scans. The patient had developed a left adrenal gland metastasis without locoregional failure. PET/CT also revealed that the metastasis was only present in the left adrenal gland (Fig. 1E). The patient subsequently received a laparoscopic left adrenalectomy; histological examination indicated poorly-differentiated squamous cell carcinoma (Fig. 2). One month following this surgery, the patient experienced abdominal pain and they were examined using an abdominal-pelvic $\mathrm{CT}$, which demonstrated the presence of multiple nodules at the left adrenalectomy site. Although the patient was provided palliative chemotherapy, they succumbed to the disease four months later.

\section{Discussion}

Malignant neoplasms arising from the paranasal sinuses are rare and the diagnosis of sinus malignancies is challenging due to the frequently nonspecific symptoms and the deep position of the structures involved, which are difficult to biopsy (5). Therefore, paranasal sinus cancer is typically diagnosed as an advanced T3/T4 stage disease (7). Nodal metastasis is experienced by $\leq 26 \%$ of patients (8). In the present case, the patient exhibited swelling of the left submandibular area and was experiencing pain, so they were prescribed a three-week course of oral antibiotics, due to what was eventually revealed to be a misdiagnosis of odontogenic sinusitis. The accurate diagnosis was obtained following a biopsy of the protruding granulation tissue at the tooth extraction site. After staging work up, the patient was determined to have a locally advanced maxillary sinus squamous cell carcinoma at stage IVA.

The optimal treatment strategy for locally advanced paranasal sinus cancer remains undefined (3). Current therapeutic approaches include surgery, radiation and systemic chemotherapy in a variety of combinations and sequences $(2,3)$. Prior studies have reported that a combination of surgery and radiotherapy can provide an improved rate of overall survival (OS), when compared with CRT, for locally advanced paranasal sinus cancer $(9,10)$. Kang et al (11) reported that a combination of surgery and radiotherapy and/or chemotherapy conferred superior five-year OS rates, as compared with CRT, for stage III or localized stage IV maxillary sinus cancer (81.2 vs. $37.9 \%$; log-rank test, $\mathrm{P}=0.029$ ), which were analyzed using SPSS software version 16.0 (SPSS, Inc., Chicago, IL, USA). The patient in the current report had received a left partial maxillectomy and ipsilateral radical neck lymph node dissection followed by postoperative CRT. The patient achieved complete remission; however, the maxillary sinus cancer rapidly progressed to the left adrenal gland after three months of CRT. In the event of isolated adrenal metastasis in lung cancer, or liver metastasis in colorectal cancer, the patients in question are considered to have stage IV non-small cell lung cancer or colorectal cancer, respectively. However, it has previously been noted that aggressive surgical treatment of the metastasis may result in long-term survival. Luketich et al (12) reported that excision significantly prolonged survival time (31 months), as compared with no excision (8.5 months), in adrenal metastasis of primary non-small cell lung cancer. In patients with colorectal cancer and liver metastasis, the five-year survival rate with chemotherapy alone is 5-10\% (13). However, liver resection in selected patients can result in a five-year survival rate of $30 \%$ (14). In the current case, a laparoscopic left adrenalectomy was performed for salvage treatment. Despite this, the maxillary sinus cancer rapidly progressed and the patient succumbed to the disease four months later.

Waldron et al (3) evaluated 110 cases of maxillary sinus cancer with definitive RT (83 patients) or surgery and adjuvant RT (27 patients). The five-year rates of local progression-free survival and disease-free survival were 42 and $43 \%$, respectively. In total, 63 patients developed local $\mathrm{r}$ ecurrence, and $25 / 63$ then underwent salvage surgery with a subsequent five-year cause-specific survival rate of $31 \%$ (3). Therefore, localized recurrence following surgery and/or CRT may be managed by salvage surgery. However, further investigations into salvage surgery for isolated distant metastasis are required.

In conclusion, it is suggested that clinicians must consider that maxillary sinus cancer may recur only at the distant site. Salvage surgery alone for isolated distant metastases is not a sufficient treatment option and postoperative radiotherapy or chemotherapy must be considered, whether or not residual tumor tissue is absent. The role of salvage surgery for the isolated metastasis of paranasal sinus cancer must be investigated further.

\section{References}

1. Ansa B, Goodman M, Ward K, Kono SA, Owonikoko TK, Higgins K, Beitler JJ, Grist W, Wadsworth T, El-Deiry M, et al: Paranasal sinus squamous cell carcinoma incidence and survival based on surveillance, epidemiology, and end results data, 1973 to 2009. Cancer 119: 2602-2610, 2013

2. Bristol IJ, Ahamad A, Garden AS, Morrison WH, Hanna EY, Papadimitrakopoulou VA, Rosenthal DI and Ang KK: Postoperative radiotherapy for maxillary sinus cancer: Long-term outcomes and toxicities of treatment. Int J Radiat Oncol Biol Phys 68: 719-730, 2007.

3. Waldron JN, O'Sullivan B, Gullane P, Witterick IJ, Liu FF, Payne D, Warde P and Cummings B: Carcinoma of the maxillary antrum: A retrospective analysis of 110 cases. Radiother Oncol 57: 167-173, 2000.

4. Edge SB, Byrd DR, Compton CC, Fritz AG, Greene FL, Trotti A: AJCC cancer staging manual. 7th edition. New York, NY, Springer, 2010.

5. Bhattacharyya N: Factors affecting survival in maxillary sinus cancer. J Oral Maxillofac Surg 61: 1016-1021, 2003.

6. Muir CS and Nectoux J: Descriptive epidemiology of malignant neoplasms of nose, nasal cavities, middle ear and accessory sinuses. Clin Otolaryngol Allied Sci 5: 195-211, 1980. 
7. Santos MR, Servato JP, Cardoso SV, de Faria PR, Eisenberg AL, Dias FL and Loyola AM: Squamous cell carcinoma at maxillary sinus: Clinicopathologic data in a single Brazilian institution with review of literature. Int J Clin Exp Pathol 7: 8823-8832, 2014.

8. Myers LL, Nussenbaum B, Bradford CR, Teknos TN, Esclamado RM and Wolf GT: Paranasal sinus malignancies: An 18-year single institution experience. Laryngoscope 112: 1964-1969, 2002.

9. Hayashi T, Nonaka S, Bandoh N, Kobayashi Y, Imada M and Harabuchi Y: Treatment outcome of maxillary sinus squamous cell carcinoma. Cancer 92: 1495-1503, 2001.

10. Dulguerov P, Jacobsen MS, Allal AS, Lehmann W and Calcaterra T: Nasal and paranasal sinus carcinoma: Are we making progress? A series of 220 patients and a systematic review. Cancer 92: 3012-3029, 2001.

11. Kang JH, Cho SH, Kim JP, Kang KM, Cho KS, Kim W, Seol YM, Lee S, Park HS, Hur WJ, et al: Treatment outcomes between concurrent chemoradiotherapy and combination of surgery, radiotherapy, and/or chemotherapy in stage III and IV maxillary sinus cancer: Multi-institutional retrospective analysis. J Oral Maxillofac Surg 70: 1717-1723, 2012.
12. Luketich JD and Burt ME: Does resection of adrenal metastases from non-small cell lung cancer improve survival? Ann Thorac Surg 62: 1614-1616, 1996

13. Gallinger S, Biagi JJ, Fletcher GG, Nhan C, Ruo L and McLeod RS: Liver resection for colorectal cancer metastases. Curr Oncol 20: e255-e265, 2013.

14. Simmonds PC, Primrose JN, Colquitt JL, Garden OJ, Poston GJ and Rees M: Surgical resection of hepatic metastases from colorectal cancer: A systematic review of published studies. Br J Cancer 94: 982-999, 2006. 